\title{
A Novel Diagnostic and Prognostic Score for Abdominal Aortic Aneurysms Based on D-Dimer and a Comprehensive Analysis of Myeloid Cell Parameters
}

\author{
Branislav Zagrapan ${ }^{1}$ Wolf Eilenberg ${ }^{1}$ Suriya Prausmueller ${ }^{1}$ Paimann Nawrozi $^{1}$ Katharina Muench ${ }^{1}$ \\ Sarah Hetzer ${ }^{1}$ Vanessa Elleder ${ }^{1}$ Renata Rajic ${ }^{1} \quad$ Felix Juster $^{1}$ Luca Martelanz ${ }^{1}$ Hubert Hayden ${ }^{1}$ \\ Johannes Klopf ${ }^{1}$ Cansu Inan ${ }^{1}$ Peter Teubenbacher ${ }^{1}$ Markus P. Weigl ${ }^{1}$ Patrick Kirchweger ${ }^{1}$ \\ Dietrich Beitzke $^{2}$ Bernd Jilma ${ }^{3}$ Johann Wojta ${ }^{4}$ Marc A. Bailey ${ }^{5,6} \quad$ D. Julian A. Scott ${ }^{6,5}$ Ihor Huk ${ }^{1}$ \\ Christoph Neumayer ${ }^{1}$ Christine Brostjan ${ }^{10}$
}

\footnotetext{
${ }^{1}$ Division of Vascular Surgery and Surgical Research Laboratories, Department of Surgery, Medical University of Vienna, Vienna General Hospital, Vienna, Austria

${ }^{2}$ Division of Cardiovascular and Interventional Radiology, Department of Biomedical Imaging and Image Guided Therapy, Medical University of Vienna, Vienna General Hospital, Vienna, Austria

3 Department of Clinical Pharmacology, Medical University of Vienna, Vienna General Hospital, Vienna, Austria

${ }^{4}$ Division of Cardiology, Department of Internal Medicine II, Medical University of Vienna, Vienna General Hospital, Vienna, Austria

${ }^{5}$ Leeds Institute of Cardiovascular and Metabolic Medicine, Faculty of Medicine and Health, University of Leeds, Leeds, United Kingdom

6 Leeds Vascular Institute, Leeds General Infirmary, Leeds,

United Kingdom
}

Thromb Haemost 2019;119:807-820.
Address for correspondence Christine Brostjan, PhD, Department of Surgery, Medical University of Vienna, Anna Spiegel Centre for Translational Research, Vienna General Hospital 25.05.002, A-1090 Vienna, Austria (e-mail: christine.brostjan@meduniwien.ac.at).

\section{Abstract \\ Keywords \\ - abdominal aortic aneurysm \\ - D-dimer \\ - diagnosis \\ - myeloperoxidase \\ - prognosis}

The pathogenesis of abdominal aortic aneurysm (AAA) involves a central component of chronic inflammation which is predominantly mediated by myeloid cells. We hypothesized that the local inflammatory activity may be reflected in systemic alterations of neutrophil and monocyte populations as well as in soluble factors of myeloid cell activation and recruitment. To establish their marker potential, neutrophil and monocyte sub-sets were measured by flow cytometry in peripheral blood samples of 41 AAA patients and 38 healthy controls matched for age, sex, body mass index and smoking habit. Comparably, circulating factors reflecting neutrophil and monocyte activation and recruitment were assayed in plasma. Significantly elevated levels of CD16+ monocytes, activated neutrophils and newly released neutrophils were recorded for AAA patients compared with controls. In line, the monocyte chemoattractant $\mathrm{C}-\mathrm{C}$ chemokine ligand 2 and myeloperoxidase were significantly increased in patients' plasma. The diagnostic value was highest for myeloperoxidase, a mediator which is released by activated neutrophils as well as CD16+ monocytes. Multivariable regression models using myeloid activation markers and routine laboratory parameters

\footnotetext{
(D) Christine Brostjan's ORCID is https://orcid.org/0000-0003-1462-

5397.
}

received

October 27, 2018

accepted after revision January 12, 2019
DOI https://doi.org/

10.1055/s-0039-1679939. ISSN 0340-6245. (c) 2019 Georg Thieme Verlag KG Stuttgart · New York
License terms

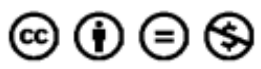


identified myeloperoxidase and D-dimer as strong independent correlates of AAA. These two biomarkers were combined to yield a diagnostic score which was subsequently challenged for confounders and confirmed in a validation cohort matched for cardiovascular disease. Importantly, the score was also found suited to predict rapid disease progression. In conclusion, D-dimer and myeloperoxidase represent two sensitive biomarkers of AAA which reflect distinct hallmarks (thrombus formation and inflammation) of the pathomechanism and, when combined, may serve as diagnostic and prognostic AAA score warranting further evaluation.

\section{Introduction}

Abdominal aortic aneurysm (AAA) represents a major cause of cardiovascular mortality despite advancements in surgical techniques. It is frequently asymptomatic, diagnosed incidentally and associated with a high death rate at rupture. ${ }^{1}$ Simple low-cost biomarkers for early disease detection would be beneficial in countries where ultrasound screening for high-risk groups is not implemented. Importantly, prognostic markers are of particular interest because rapid expansion of small aneurysms is difficult to predict but associated with a high risk of rupture. ${ }^{1}$ Blood-borne diagnostic parameters which have repeatedly shown association with AAA are common biomarkers of cardiovascular disease such as D-dimer, fibrinogen ${ }^{2,3}$ or C-reactive protein. ${ }^{4}$ Among these, D-dimer was reported to be related to the occurrence of aortic aneurysm independent of underlying atherosclerotic disease, ${ }^{5}$ to correlate with AAA and intraluminal thrombus (ILT) size $^{6}$ and to predict aneurysm growth. ${ }^{7,8}$

Histologically, the AAA wall is characterized by destruction of the vascular media associated with loss of elastic fibres and smooth muscle cells (SMCs). ${ }^{9}$ Prominent inflammatory infiltrates in the adventitia as well as the ILT exert wall-weakening activity which is primarily attributed to myeloid cells and their production of reactive oxygen species (ROS) and proteolytic enzymes. ${ }^{10}$ Several substances in the ILT and AAA wall which originate from activated neutrophils and monocytes can be detected at increased levels in the peripheral blood of AAA patients and have been investigated for their biomarker potential. These include: extracellular matrix-degrading enzymes such as cathepsins, ${ }^{11}$ matrix metalloproteinases, elastase, ${ }^{10}$ neutrophil gelatinase-associated lipocalin ${ }^{12}$ and inflammatory cytokines. ${ }^{13}$ In contrast to these soluble factors, phenotypic alterations of the circulating myeloid cell populations are largely unexplored in the context of AAA. Monocytes form functionally distinct subsets that are recognized based on their differential surface expression of $\mathrm{CD} 14$ and $\mathrm{CD} 16 .{ }^{14}$ There is a progressive differentiation from classical (CD14++ CD16-) via intermediate $(\mathrm{CD} 14++\mathrm{CD} 16+)$ to non-classical (CD14+ CD16++) monocytes. ${ }^{15}$ Classical monocytes were identified as potent phagocytic cells ${ }^{16,17}$ with the capacity to downregulate the pro-inflammatory reactions of the two CD16+ sub-sets. ${ }^{18}$ The non-classical population exhibited patrolling behaviour maintaining vessel integrity, ${ }^{19,20}$ while inter- mediate monocytes were proposed to have the highest capacity for antigen presentation, angiogenesis and tissue remodelling. ${ }^{16,17}$ Furthermore, intermediates showed the highest propensity to form monocyte-platelet aggregates (MPAs), which are of relevance to thrombotic disease. ${ }^{21}$ Increased levels of intermediate and non-classical monocytes were found to be associated with a higher risk of cardiovascular events $^{22}$ as well as large AAAs. ${ }^{23,24}$

Comparably, the existence of neutrophil sub-sets with distinct activation status and function has been proposed which are defined by the expression of CD62L and CD16. ${ }^{25}$ Mature, quiescent circulating neutrophils are generally characterized by a high level of both surface markers (CD16 ${ }^{\text {high }}$ CD62L $\left.{ }^{\text {high }}\right)$. The immature $\mathrm{CD} 16^{\text {low }} \mathrm{CD} 62 \mathrm{~L}^{\text {high }}$ neutrophil sub-set which is newly released from bone marrow is marked by lower phagocytic activity and decreased ROSgenerating capability, while the activated $\mathrm{CD} 16^{\text {high }} \mathrm{CD} 62 \mathrm{~L}^{\text {low }}$ sub-set is strongly pro-inflammatory and exerts main immunoregulatory functions. ${ }^{25,26}$ Both, activated as well as newly released sub-sets are induced in endotoxaemia and sepsis, ${ }^{25}$ but relevant studies in other disease-specific contexts, including AAA, are lacking. However, the interaction of neutrophils with platelets has been shown to play an important role in AAA pathogenesis, enhancing neutrophil recruitment and activation. ${ }^{10}$

Based on the reports of neutrophil and monocyte involvement in AAA, we postulated that the state of chronic inflammation is not restricted to the local AAA site but is reflected in a systemic change in myeloid cell populations indicating on-going leukocyte activation and recruitment. We thus assessed the marker potential of circulating myeloid cell sub-sets and their secreted factors in comparison to previously reported AAA parameters such as D-dimer. A diagnostic risk score was devised and challenged in a validation cohort. Of particular interest, the risk score further proved effective in predicting rapid disease progression.

\section{Methods}

\section{Diagnostic Study Design}

We conducted an observational case-control study, adhering to the principles of the Declaration of Helsinki and the STROBE guidelines for reporting results. The study was approved by the institutional ethics committee (license no. 1729/2014) and informed consent was obtained from 
participating patients at the Vienna General Hospital (20142016). Morphometric AAA analysis was performed using computed tomography angiography (CTA) images at study inclusion. A control group was recruited from general surgery, urology and ophthalmology patients (presenting for routine check-ups) with ultrasound-confirmed absence of AAA. Subjects with recent ( $<1$ year) tumour and/or chemotherapy, systemic autoimmune or haematological disease and organ transplant recipients were excluded. In the present evaluation, data collected from 41 patients prior to immanent elective surgical repair via open or endovascular approach were compared with the control group $(N=38)$, which was matched in age, sex, body mass index and smoking habit.

For validation of the diagnostic score, citrated plasma samples collected at an independent institution (Leeds General Infirmary as part of the Leeds Aneurysm Development Study, approved by the Leeds East Ethics Committee, Ref 03/ $142^{27-31}$ ) were analysed which represented 63 AAA cases and 63 controls, recruited from other medical and surgical outpatient departments at the same hospital. All patients received abdominal aortic ultrasound for confirmation of diagnosis. For these samples, one-to-one matching was performed for age (within 2 years), sex and the presence of clinical cardiovascular disease (previous myocardial infarction, angina pectoris, peripheral vascular disease or cerebrovascular disease).

\section{Prognostic Study Design}

Based on the mentioned inclusion and exclusion criteria, AAA patients without surgical indication were enrolled in a longitudinal observational study (2014-2018) at the Vascular Surgery Department of the Medical University of Vienna, with serial blood withdrawing and CTA analysis every 6 months. Thirty-three patients were assessed, with a total of 68 six-month monitoring periods.

\section{Flow Cytometry of Myeloid Cell Populations}

Blood was collected into hirudin containing tubes (Roche, Basel, Switzerland) and fixed immediately with ThromboFix solution (Beckman-Coulter, Indianapolis, Indiana, United States) according to manufacturer's instructions. After 2 to 7 hours at room temperature, leukocyte populations were stained in whole blood using the following antibodies: CD66b-FITC, CD62L-AF647, CD16-PE/Cy7, CD41-PB (BioLegend, San Diego, California, United States) and CD54-PE (Thermo Fisher Scientific, Waltham, Massachusetts, United States) for neutrophil sub-sets; CD14-FITC, CD16-PE/Cy7, HLADR-PE and CD41-PB (BioLegend) for monocyte sub-sets. 7AAD dye (Beckman-Coulter) was used to exclude non-viable cells. Following erythrocyte lysis with RBC Lysis Buffer (Thermo Fisher Scientific), samples were diluted and immediately analysed with a Gallios flow cytometer (BeckmanCoulter). Neutrophil sub-sets were evaluated as viable $\mathrm{CD66b}+$ cells and distinguished from eosinophils (CD16-). Sub-set discrimination was based on CD62L and CD16 expression levels (- Supplementary Figs. S1 and S2, available in the online version), identifying mature, quiescent neutrophils as
$\mathrm{CD} 16^{\text {high }} \mathrm{CD} 62 \mathrm{~L}^{\text {high }}$, and the activated neutrophil population as $\mathrm{CD} 16^{\text {high }} \mathrm{CD} 62 \mathrm{~L}^{\text {low }}$ as opposed to newly released neutrophils with $\mathrm{CD} 16^{\text {low }}$ CD62 $\mathrm{L}^{\text {high }}$ surface expression. Monocyte subsets were quantified from viable HLA-DR + CD14+ cells using CD14 and CD16 surface levels (-Supplementary Fig. S3, available in the online version) for the distinction between classical (CD14++ CD16-), intermediate (CD14++ CD16+) and non-classical (CD14+ CD16++) monocytes. Both, neutrophils and monocytes were additionally analysed for the proportion of platelet (CD41+) aggregates. A blood count was performed using the same hirudin-treated blood sample on a Sysmex XN-350 haemocytometer (Sysmex, Kobe, Japan) which was applied to calculate absolute concentrations of leukocyte sub-sets.

Since standard methods were applied to measure plasma and tissue-released factors, the respective methods are described in the -Supplementary Methods (available in the online version).

\section{Statistical Evaluation}

Data are generally given as median values and interquartile range (IQR). Non-parametric tests were used for group comparisons (Mann-Whitney U, Wilcoxon signed-rank test) or correlations (Spearman's $r$ ). Contingency tables and chi-square test were applied for categorical variables. Receiver operating characteristics (ROC) analysis served to evaluate the diagnostic or prognostic marker potential. These analyses were performed with SPSS 24.0 software (IBM, Armonk, New York, United States) and a significance level of $p<0.05$ was applied. Sample size calculation was based on the previously reported frequency of CD16+ monocytes in AAA patients and healthy controls ${ }^{23}$ with a 1.4 -fold difference in mean parameter levels at a high biological variation $(\mu 1=11.0 \%, \mu 2=15.1 \%, \sigma=6.1 \%, \alpha=0.05,1-\beta=$ 0.80 ) and yielded a minimal sample size of 36 per group. Missing values were generally below $5 \%$ and hence not addressed in statistical analysis.

Logistic regression models were developed with $\mathrm{R}$ software (R Core Team 2016, Vienna, Austria) using stepwise backward elimination of insignificant variables and the Akaike information criterion as the stopping criterion. The resulting diagnostic model was internally validated, using fivefold cross-validated predicted probabilities. The validation was repeated 100 times and results were averaged to avoid dependence on a specific data split. The area under the ROC curve was deduced from ROC analyses comparing the predicted probabilities and the observed event status. Finally, a coefficient of discrimination ${ }^{32}$ was computed as the difference in average (cross-validated) predicted probabilities between patients diagnosed with AAA and individuals without AAA.

The developed diagnostic score was further evaluated in the original dataset by multivariable binary logistic regression including co-morbidities and patient medication as categorical variables: coronary heart disease (CHD), antiplatelet therapy, hypertension and/or anti-hypertensive therapy, hyperlipidaemia and/or lipid-lowering therapy. (Due to the inherent correlation of hypertension and 
hyperlipidaemia with their pertaining therapies, the combined variables were applied.) The score was further characterized in the above-mentioned, independent validation set by ROC curve analysis (SPSS 24.0).

\section{Results}

Patient and Control Group Differ Significantly in the Reported Haematological Biomarker D-dimer, in AAAAssociated Co-Morbidities and Medication

Forty-one AAA patients and 38 control subjects were included in the analysis. Groups were comparable in terms of: sex distribution, age, smoking status and body mass index (-Tables 1, 2, 3). There were significant differences in the diagnosis of hypertension $(p=0.026)$ and prescription of anti-hypertensive therapy $(p=0.051)$, which were more frequent in the AAA cohort. More aneurysm patients than controls were affected by hyperlipidaemia $(p=0.001)$ and under lipid-lowering treatment $(p<0.001)$. The AAA group

Table 1 Patient and control demographics: categorical variables

\begin{tabular}{|c|c|c|c|}
\hline Characteristic & Healthy $(N=38)$ & AAA $(N=41)$ & $p$-Value \\
\hline & $N(\%)$ & $N(\%)$ & \\
\hline \multicolumn{3}{|l|}{ Sex } & \multirow[t]{3}{*}{0.390} \\
\hline Female & $5(13.2)$ & $3(7.3)$ & \\
\hline Male & $33(86.8)$ & $38(92.7)$ & \\
\hline \multicolumn{4}{|l|}{ Smoker status } \\
\hline Never & $8(21.1)$ & $4(9.8)$ & \multirow[t]{3}{*}{0.160} \\
\hline Past & $13(34.2)$ & $22(53.7)$ & \\
\hline Current & $16(42.1)$ & $14(34.1)$ & \\
\hline Unknown & $1(2.6)$ & $1(2.4)$ & \\
\hline Hypertension & $23(60.5)$ & 34 (82.9) & 0.026 \\
\hline Hyperlipidaemia & $11(28.9)$ & $27(65.9)$ & 0.001 \\
\hline \multicolumn{4}{|l|}{$\begin{array}{l}\text { Peripheral artery } \\
\text { disease }\end{array}$} \\
\hline No & $34(89.5)$ & $32(78.0)$ & \multirow[t]{3}{*}{0.250} \\
\hline Yes & $4(10.5)$ & $7(17.1)$ & \\
\hline Unknown & $0(0.0)$ & $2(4.9)$ & \\
\hline $\begin{array}{l}\text { Coronary heart } \\
\text { disease }\end{array}$ & $4(10.5)$ & $14(34.1)$ & 0.012 \\
\hline Myocardial infarction & $3(7.9)$ & $9(22.0)$ & 0.082 \\
\hline Stroke & $0(0.0)$ & $3(7.3)$ & 0.236 \\
\hline Diabetes mellitus & $4(10.5)$ & $9(22.0)$ & 0.171 \\
\hline COPD & $5(13.2)$ & $13(31.7)$ & 0.050 \\
\hline \multicolumn{4}{|l|}{ Nephropathy/renal cysts } \\
\hline No & $31(81.6)$ & $27(65.9)$ & \multirow[t]{3}{*}{0.227} \\
\hline Yes & $7(18.4)$ & $13(31.7)$ & \\
\hline Unknown & $0(0.0)$ & $1(2.4)$ & \\
\hline \multicolumn{4}{|l|}{ AAA family history } \\
\hline No & 34 (89.5) & 34 (82.9) & \multirow[t]{3}{*}{0.236} \\
\hline Yes & $4(10.5)$ & $4(9.8)$ & \\
\hline Unknown & $0(0.0)$ & $3(7.3)$ & \\
\hline
\end{tabular}

Abbreviations: AAA, abdominal aortic aneurysm; COPD, chronic obstructive pulmonary disease. had lower total cholesterol $(p=0.037)$ and high-density lipoprotein $(p=0.030)$. The groups further differed significantly in the occurrence of CHD $(p=0.012)$, with AAA patients having an almost threefold higher frequency of anti-platelet drug use $(p<0.001)$. A higher cardiovascular burden in the AAA group was also evidenced by the Framingham Risk Score in per cent 10 -year risk of general cardiovascular disease $(p=0.026)$. Other co-morbidities such as chronic obstructive pulmonary disease and nephropathy (mainly renal cysts) showed a trend but no significant elevation in the disease collective. Nevertheless, kidney parameters indicated a poorer kidney function in AAA patients: elevated blood urea nitrogen $(p=0.001)$ and creatinine ( $p=0.059)$ and reduced estimated glomerular filtration rate $(p=0.045)$.

In the AAA patient group, the median maximum AAA diameter reached $57 \mathrm{~mm}$ (range: $44-114 \mathrm{~mm}$ ) with a median aneurysm length of $92 \mathrm{~mm}$ (38-147 mm). Regarding the ILT, a median maximum thickness of $22 \mathrm{~mm}(5-45 \mathrm{~mm})$ and volume of $75 \mathrm{~mm}^{3}\left(2-347 \mathrm{~mm}^{3}\right.$ ) was recorded (-Table 4). Only 5 of 41 patients showed a concomitant thoracic aortic aneurysm; the AAA form was predominantly fusiform (63\%).

In line with published studies, ${ }^{3}$ the plasma D-dimer concentration (-Table 2) was highly increased in AAA patients compared with controls (median: $1.30 \mu \mathrm{g} / \mathrm{mL}$ [IQR $=1.68]$ vs. $0.47 \mu \mathrm{g} / \mathrm{mL}$ [IQR $=0.49], p<0.001)$ and correlated with AAA maximum diameter $(r=0.537, p=0.001)$ and ILT maximum diameter $(r=0.500, p=0.009)$.

\section{AAA Patients Show a Higher Frequency of Activated and Newly Released Neutrophils as well as CD16+ Monocytes}

The study focused on evaluating possible alterations in the distribution of circulating neutrophil and monocyte sub-sets in AAA patients compared with controls. Activated neutrophils, marked by the loss of CD62L fluorescent signal intensity (- Supplementary Figs. S1 and \$2, available in the online version), were significantly more prevalent in the AAA patient group (-Table 5). The median number of activated neutrophils in AAA patients ( - Fig. 1C) was $31 \times 10^{6}$ cells $/ \mathrm{L}$ $(\mathrm{IQR}=24)$, while it was $21 \times 10^{6}$ cells/L $(\mathrm{IQR}=16)$ in the healthy group, $p=0.005$. Regarding newly released neutrophils (- Fig. 1B), AAA patients had $38 \times 10^{6}$ cells/L (IQR $=31)$ versus $31 \times 10^{6}$ cells/L $(\mathrm{IQR}=23)$ in the healthy control group, $p=0.020$. A significant correlation was found between the absolute concentration of circulating activated and newly released neutrophils (Spearman's $r=0.584$, $p<0.001,-$ Fig. 1F). The concentration of total neutrophils (-Fig. 1A), the mature, quiescent sub-set (-Fig. 1D) and neutrophil-platelet aggregates (-Fig. 1E) did not differ significantly between the two groups.

Our analysis further revealed a higher median frequency of CD16+ circulating monocytes of $18.8 \%(I Q R=10.0)$ in the AAA patient group compared with $16.0 \%(\mathrm{IQR}=10.7)$ in the control collective, $p=0.039$ ( - Table 5 , - Fig. 2F). The ratio of CD16-/CD16+ monocytes was calculated as a measure of monocyte activation and was significantly changed in AAA patients with a median value of $4.33(\mathrm{IQR}=2.73$ ) versus 5.24 
Table 2 Patient and control demographics: metric variables

\begin{tabular}{|c|c|c|c|}
\hline Characteristic & Healthy & AAA & $p$-Value \\
\hline & Median (Range) & Median (Range) & \\
\hline Age $[y]$ & $67(39-83)$ & $71(52-83)$ & 0.705 \\
\hline \multirow[t]{2}{*}{ Body mass index $\left[\mathrm{kg} / \mathrm{m}^{2}\right]$} & $26.2(20.7-40.1)$ & $28.4(17.8-34.1)$ & 0.370 \\
\hline & Median (IQR) & Median (IQR) & \\
\hline White blood cells $\left[\times 10^{6} / \mathrm{L}\right]$ & $5650(2100)$ & $6400(2190)$ & 0.114 \\
\hline Red blood cells $\left[\times 10^{12} / \mathrm{L}\right]$ & $4.70(0.68)$ & $4.52(0.73)$ & 0.441 \\
\hline Haemoglobin $[\mathrm{g} / \mathrm{dL}]$ & $14.2(2.7)$ & $14.6(2.4)$ & 0.543 \\
\hline Haematocrit [\%] & $41.6(6.1)$ & $41.5(6.7)$ & 0.669 \\
\hline Platelets $\left[\times 10^{9} / \mathrm{L}\right]$ & $178.5(91)$ & $157(80)$ & 0.101 \\
\hline Lymphocytes $\left[\times 10^{6} / L\right]$ & $1641(818)$ & 1611 (989) & 0.322 \\
\hline Monocytes $\left[\times 10^{6} / \mathrm{L}\right]$ & $518(292)$ & $497(356)$ & 0.928 \\
\hline Neutrophils $\left[\times 10^{6} / \mathrm{L}\right]$ & $3375(1535)$ & $3742(1350)$ & 0.217 \\
\hline C-reactive protein [mg/dL] & $0.26(0.46)$ & $0.40(0.43)$ & 0.075 \\
\hline Fibrinogen - Clauss [mg/dL] & $375(90)$ & $396(143)$ & 0.258 \\
\hline D-dimer $[\mu \mathrm{g} / \mathrm{mL}]$ & $0.47(0.49)$ & $1.30(1.68)$ & $<0.001$ \\
\hline Lipoprotein (a) [nmol/L] & $20.0(39.0)$ & $19.5(115.0)$ & 0.482 \\
\hline Triglycerides [mg/dL] & $121(73)$ & $141(59)$ & 0.070 \\
\hline Total cholesterol [mg/dL] & $198(23)$ & $170(60)$ & 0.037 \\
\hline LDL cholesterol [mg/dL] & $119(47)$ & $93(71)$ & 0.171 \\
\hline HDL cholesterol [mg/dL] & $54(25)$ & $50(17)$ & 0.030 \\
\hline Total cholesterol/HDL ratio & $3.6(2.3)$ & $3.6(1.4)$ & 0.697 \\
\hline LDL/HDL ratio & $2.2(1.7)$ & $2.0(1.4)$ & 0.996 \\
\hline FRS [risk factors] & $0.86(1.37)$ & $1.13(0.95)$ & 0.064 \\
\hline FRS [\%] & $22.9(23.8)$ & $30.4(25.7)$ & 0.026 \\
\hline Creatinine $[\mu \mathrm{g} / \mathrm{dL}]$ & $0.94(0.22)$ & $1.02(0.45)$ & 0.059 \\
\hline Blood urea nitrogen [mg/dL] & $15.1(4.1)$ & $17.8(6.4)$ & 0.001 \\
\hline eGFR $\left[\mathrm{mL} / \mathrm{min} / 1.73 \mathrm{~m}^{2}\right]$ & $84.7(20.0)$ & $76.0(38.7)$ & 0.045 \\
\hline
\end{tabular}

Abbreviations: AAA, abdominal aortic aneurysm; eGFR, estimated glomerular filtration rate; FRS, Framingham Risk Score; HDL, high-density lipoprotein; IQR, interquartile range; LDL, low-density lipoprotein.

$(\mathrm{IQR}=4.80)$ in control individuals $(p=0.039)$. The absolute number of monocytes as well as the three respective sub-sets did not differ significantly between groups (-Fig. 2A-D). Furthermore, the blood level of MPAs was not significantly different between patients and controls (-Fig. 2E).

\section{Plasma Factors Related to Myeloid Cell Activation and Recruitment are Elevated in AAA Patients and Plasma Myeloperoxidase Correlates with Tissue-Released Protein from Aneurysm Wall and ILT}

We screened plasma samples from 10 AAA patients and 10 control subjects using a multi-cytokine panel (myeloperoxidase [MPO], eotaxin, macrophage inflammatory protein [MIP]- $1 \alpha, \quad$ growth-regulated oncogene- $\alpha$, interferon gamma-inducible protein-10, interleukin-8, C-C chemokine ligand 2 [CCL2], MIP-1 $1 \beta$, RANTES and stromal cell-derived factor- $1 \alpha$ ). Only plasma levels of MPO and CCL2 (also termed monocyte chemoattractant protein 1 ) were significantly increased in the AAA patients in the pilot analysis, so MPO and CCL2 were prioritized for assessment in the entire cohort. This difference was confirmed using enzyme-linked immunosorbent assay (ELISA) for the total sample set. The median CCL2 chemokine concentration ( - Fig. 3A) in blood of AAA patients was $183 \mathrm{pg} / \mathrm{mL}(\mathrm{IQR}=45)$ as compared with $170 \mathrm{pg} / \mathrm{mL}(\mathrm{IQR}=62)$ in the healthy controls, $p=0.048$. Of note, the soluble MPO level (-Fig. 3B) was almost twice as high among AAA patients compared with control subjects (median: $13.3 \mathrm{ng} / \mathrm{mL}$ [IQR $=13.1]$ vs. $7.7 \mathrm{ng} / \mathrm{mL}$ [IQR = 3.6], $p<0.001$ ).

Based on the robust association of plasma MPO level with AAA status, we investigated conditioned media from the AAA wall $(N=13)$ to see if the potential source of MPO was indeed the AAA site. Compared with healthy aortic tissue $(N=13)$, more MPO was released from AAA wall samples (median: $639 \mathrm{ng} / \mathrm{mL}$ [IQR $=401]$ vs. $56 \mathrm{ng} / \mathrm{mL}$ [IQR $=277]$, $p<0.001)$. Of note, there was a linear correlation between 
812 Novel Diagnostic and Prognostic AAA Score Zagrapan et al.

Table 3 Patient and control medication

\begin{tabular}{|c|c|c|c|}
\hline Characteristic & Healthy $(N=38)$ & AAA $(N=41)$ & $p$-Value \\
\hline & $N(\%)$ & $N(\%)$ & \\
\hline Anti-platelet therapy & $12(31.6)$ & $36(87.8)$ & $<0.001$ \\
\hline Acetylsalicylic acid & $11(28.9)$ & $36(87.8)$ & $<0.001$ \\
\hline Clopidogrel & $1(2.6)$ & $3(7.3)$ & 0.343 \\
\hline Prasugrel or Ticagrelor & $0(0.0)$ & $2(4.9)$ & 0.386 \\
\hline Anticoagulation therapy & $6(15.8)$ & $9(22.0)$ & 0.485 \\
\hline Anti-hypertensive therapy & $23(60.5)$ & $33(80.5)$ & 0.051 \\
\hline ACE inhibitor & $5(13.2)$ & $12(29.3)$ & 0.082 \\
\hline Angiotensin receptor blocker & $10(26.3)$ & $16(39.0)$ & 0.230 \\
\hline Beta blocker & $11(28.9)$ & $21(51.2)$ & 0.044 \\
\hline Calcium channel blocker & $7(18.4)$ & $11(26.8)$ & 0.373 \\
\hline Nitrate & $0(0.0)$ & $1(2.4)$ & 0.333 \\
\hline Diuretic & $8(21.1)$ & $16(39.0)$ & 0.083 \\
\hline Lipid-lowering therapy & $6(15.8)$ & $34(82.9)$ & $<0.001$ \\
\hline Statins & $6(15.8)$ & $33(80.5)$ & $<0.001$ \\
\hline Diabetic medication & $4(10.5)$ & $8(19.5)$ & 0.266 \\
\hline Insulin & $1(2.6)$ & $1(2.4)$ & 0.957 \\
\hline Metformin & $3(7.9)$ & $7(17.1)$ & 0.220 \\
\hline Hormones & $3(7.9)$ & $6(14.6)$ & 0.346 \\
\hline Gout medication & $4(10.5)$ & $6(14.6)$ & 0.583 \\
\hline
\end{tabular}

Abbreviations: AAA, abdominal aortic aneurysm; ACE, angiotensin-converting enzyme.

Table 4 Aneurysm morphology

\begin{tabular}{|l|l|l|}
\hline Characteristic & Median & Range \\
\hline AAA maximum diameter [mm] & 56.7 & $44.0-114.3$ \\
\hline AAA length [mm] & 92.3 & $37.5-147.0$ \\
\hline ILT maximum diameter [mm] & 21.6 & $4.8-45.3$ \\
\hline ILT volume [mm $\left.{ }^{3}\right]$ & 75 & $2-347$ \\
\hline Characteristic & $N$ & $\%$ \\
\hline Aneurysm type & & \\
\hline AAA only & 36 & 87.8 \\
\hline AAA and TAA & 5 & 12.2 \\
\hline AAA form & & \\
\hline Saccular & 9 & 22.0 \\
\hline Fusiform & 26 & 63.4 \\
\hline Unknown & 6 & 14.6 \\
\hline Circumference [\%] & & \\
\hline$<25$ & 2 & 4.9 \\
\hline 50 & 3 & 7.3 \\
\hline 75 & 13 & 31.7 \\
\hline 100 & 15 & 36.6 \\
\hline Unknown & 8 & 19.5 \\
\hline
\end{tabular}

Thrombosis and Haemostasis Vol. 119 No. 5/2019
Table 4 (Continued)

\begin{tabular}{|l|l|l|}
\hline Characteristic & N & $\%$ \\
\hline ILT presence & & \\
\hline Yes & 33 & 80.5 \\
\hline Unknown & 8 & 19.5 \\
\hline ILT coverage & & \\
\hline Circular & 15 & 36.6 \\
\hline Partial & 17 & 41.5 \\
\hline Unknown & 9 & 22.0 \\
\hline
\end{tabular}

Abbreviations: AAA, abdominal aortic aneurysm; ILT, intraluminal thrombus; TAA, thoracic aortic aneurysm.

plasma and tissue-released MPO of patients whose aortic wall had been analysed (Spearman's coefficient $r=0.620$, $p<0.001$; - Fig. 3C and D). Furthermore, tissue-released and plasma MPO levels correlated significantly with AAA maximum diameter: $r=0.566, p=0.044$ and $r=0.391$, $p=0.013$, respectively ( $\mathbf{- F i g . ~} \mathbf{3 E}$ and $\mathbf{F}$ ).

\section{Plasma Myeloperoxidase and D-dimer Levels are Independently Associated with AAA Status and may be Combined to Yield an Advanced Diagnostic Score}

Next, a ROC analysis was performed using explorative variables which have shown significant differences between the 
Table 5 Neutrophil and monocyte sub-set frequencies in peripheral blood of AAA patients and healthy control individuals

\begin{tabular}{|c|c|c|c|}
\hline Parameter & Healthy & AAA & $p$-Value \\
\hline Neutrophil populations & Median (IQR) & Median (IQR) & \\
\hline Total neutrophils $\left[\times 10^{6} / \mathrm{L}\right]$ & $3,375(1,535)$ & $3,742(1,351)$ & 0.217 \\
\hline Quiescent neutrophils $\left[\times 10^{6} / \mathrm{L}\right]$ & $3,063(1,400)$ & $3,627(1,344)$ & 0.188 \\
\hline Newly released neutrophils $\left[\times 10^{6} / \mathrm{L}\right]$ & $31(23)$ & $38(31)$ & 0.020 \\
\hline Activated neutrophils $\left[\times 10^{6} / \mathrm{L}\right]$ & $21(16)$ & $31(24)$ & 0.005 \\
\hline Neutrophil-platelet aggregates $\left[\times 10^{6} / \mathrm{L}\right]$ & $344(410)$ & $401(562)$ & 0.617 \\
\hline Quiescent neutrophils [\%] & $98.37(0.99)$ & $98.13(1.20)$ & 0.030 \\
\hline Newly released neutrophils [\%] & $0.92(0.68)$ & $1.09(0.64)$ & 0.079 \\
\hline Activated neutrophils [\%] & $0.62(0.46)$ & $0.87(0.59)$ & 0.030 \\
\hline Neutrophil-platelet aggregates [\%] & $11.10(7.30)$ & $12.40(14.62)$ & 0.737 \\
\hline Monocyte populations & Median (IQR) & Median (IQR) & \\
\hline Total monocytes $\left[\times 10^{6} / \mathrm{L}\right]$ & $518(292)$ & $497(356)$ & 0.928 \\
\hline Classical monocytes $\left[\times 10^{6} / \mathrm{L}\right]$ & $441(277)$ & $366(256)$ & 0.506 \\
\hline Intermediate monocytes $\left[\times 10^{6} / \mathrm{L}\right]$ & $31(41)$ & $50(48)$ & 0.259 \\
\hline Non-classical monocytes $\left[\times 10^{6} / \mathrm{L}\right]$ & $38(44)$ & $49(51)$ & 0.315 \\
\hline CD16+ monocytes $\left[\times 10^{6} / L\right]$ & $73(70)$ & $109(91)$ & 0.219 \\
\hline Monocyte-platelet aggregates $\left[\times 10^{6} / \mathrm{L}\right]$ & $74(73)$ & $82(79)$ & 0.970 \\
\hline CD16-/CD16+ monocyte ratio & $5.24(4.80)$ & $4.33(2.73)$ & 0.039 \\
\hline Classical monocytes [\%] & $83.97(10.65)$ & $81.22(10.02)$ & 0.039 \\
\hline Intermediate monocytes [\%] & $7.12(4.72)$ & $7.86(3.79)$ & 0.116 \\
\hline Non-classical monocytes [\%] & $7.21(6.76)$ & $9.99(8.50)$ & 0.060 \\
\hline CD16+ monocytes [\%] & $16.03(10.65)$ & $18.78(10.02)$ & 0.039 \\
\hline Monocyte-platelet aggregates [\%] & $14.34(6.94)$ & $16.09(16.10)$ & 0.888 \\
\hline
\end{tabular}

Abbreviations: AAA, abdominal aortic aneurysm; IQR, interquartile range.

subject collectives. Of these, the highest diagnostic marker potential was seen with plasma MPO levels showing an area under the curve (AUC) of $0.816, p<0.001$, as compared with activated and newly released neutrophils (AUC $=0.684$, $p=0.005$ and AUC $=0.654, p=0.020$, respectively), \% CD16 + monocytes (AUC $=0.646, p=0.039$ ) as well as CCL2 (AUC $=0.631, p=0.048)$.

A diagnostic logistic regression model was developed including the following parameters: blood counts of total neutrophils, quiescent neutrophils, activated neutrophils, newly released neutrophils, total monocytes, the percentage of CD16+ monocytes as well as plasma levels of D-dimer, MPO and CCL2. After backward elimination, only D-dimer (odds ratio: 3.96 and 95\% confidence interval [95\% CI]: 1.6512.81) and MPO (odds ratio: 1.23, 95\% CI: 1.08-1.43) had significant independent importance for AAA diagnosis and were retained in the model. From the logistic model, a linear score was derived, according to the estimated regression coefficients, as 'score' $=-3.442+1.375^{*} \mathrm{D}$-dimer +0.205 * MPO. When compared with ROC analysis for MPO (AUC $=0.816$ ) and D-dimer (AUC $=0.830$ ) alone, the combined score performed better (AUC $=0.877$ ) in discriminating patients from healthy individuals (-Fig. 4A). Evaluation of the dichotomized score (grouped into positive and negative values) revealed a diagnostic sensitivity of $73 \%$ and specificity of $80 \%$. The score also showed a higher coefficient of correlation $(r=0.664, p<0.001)$ than MPO $(r=0.391$, $p=0.013)$ and $D$-dimer $(r=0.537, p=0.001)$ for maximal AAA diameter (-Table 6), while D-dimer $(r=0.687$, $p<0.001$ ) was more closely correlated with ILT volume than the calculated diagnostic score $(r=0.450, p=0.031)$.

\section{Myeloid Cell Parameters are Sensitive to Confounders but the Diagnostic Score Identifies AAA Irrespective of Co-Morbidities and Patient Medication}

It was further of interest to evaluate whether the significant difference in explorative parameters between AAA patients and healthy controls was lost when the collectives were matched for the presence or absence (retaining the larger sample size) of a particular co-morbidity or medication (-Supplementary Tables S1-S5, available in the online version). The blood populations of activated and newly released neutrophils were influenced by hyperlipidaemia and/or statin therapy. Similarly, when the collectives were matched for aspirin intake, the difference in neutrophil sub-set frequency was lost. Hypertension and anti-hypertensive drugs 

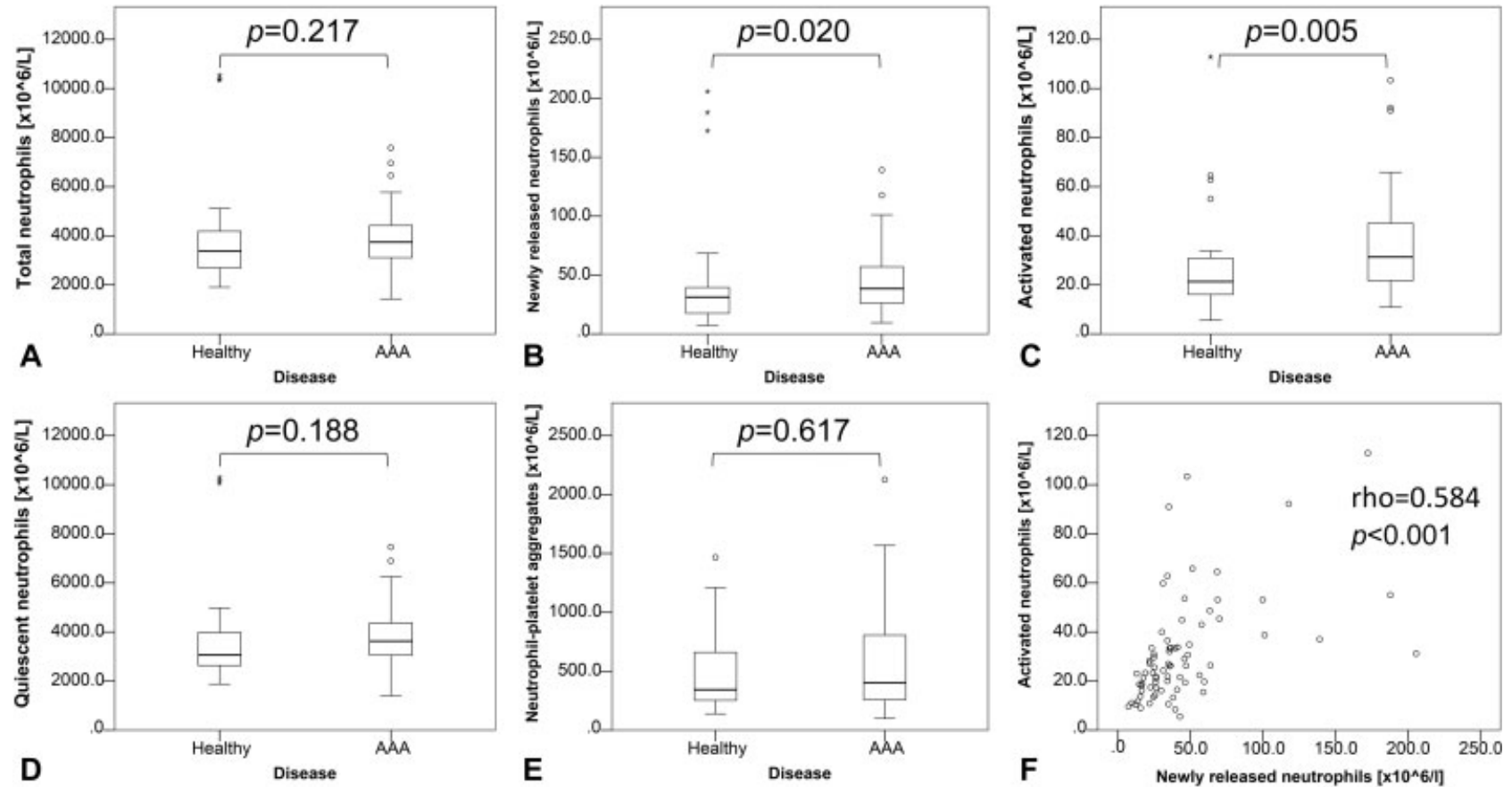

Fig. 1 Distribution of neutrophil populations in peripheral blood of abdominal aortic aneurysm (AAA) patients and healthy controls. Boxplots illustrate the absolute concentration of (A) total neutrophils, (B) the newly released, (C) activated or (D) mature, quiescent neutrophil sub-set as well as (E) neutrophil-platelet aggregates. Group comparisons were performed by Mann-Whitney $U$ test. (F) The linear correlation between activated and newly released neutrophils is evaluated by scattergram and Spearman's coefficient of correlation.
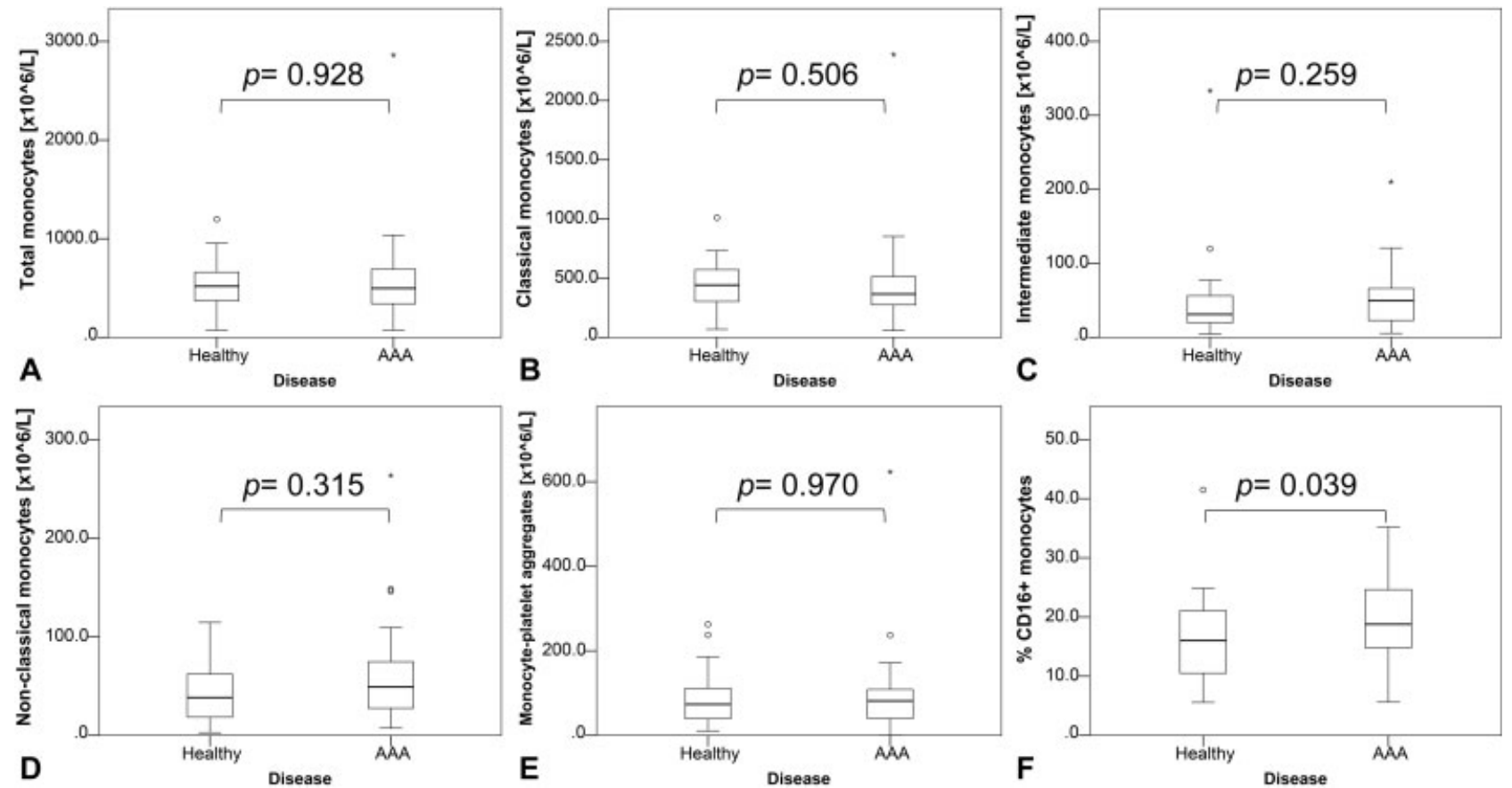

Fig. 2 Distribution of monocyte populations in peripheral blood of abdominal aortic aneurysm (AAA) patients and healthy controls. Boxplots illustrate the absolute concentration of (A) total monocytes, (B) the classical, (C) intermediate or (D) non-classical monocyte sub-set as well as (E) monocyte-platelet aggregates. Intermediate and non-classical monocytes were further combined to the group of CD16+ monocytes, and their relative frequency is shown in (F). Group comparisons were performed by Mann-Whitney U test. 



Fig. 3 Increased plasma and tissue levels of soluble factors associated with myeloid cell activation. The boxplot comparison illustrates the distribution of (A) plasma C-C chemokine ligand 2 (CCL2), (B) plasma myeloperoxidase (MPO) and (C) tissue MPO concentrations as measured by enzyme-linked immunosorbent assay (ELISA) in samples of abdominal aortic aneurysm (AAA) patients and healthy controls. For tissue analysis, sections from healthy and AAA aortic walls were incubated in medium overnight to release tissue-contained MPO. Group comparisons were performed by Mann-Whitney $U$ test. Correlations between (D) plasma and tissue-released MPO, as well as maximum AAA diameter and (E) tissue MPO or (F) plasma MPO levels are shown in scattergrams (Spearman's coefficient of correlation).
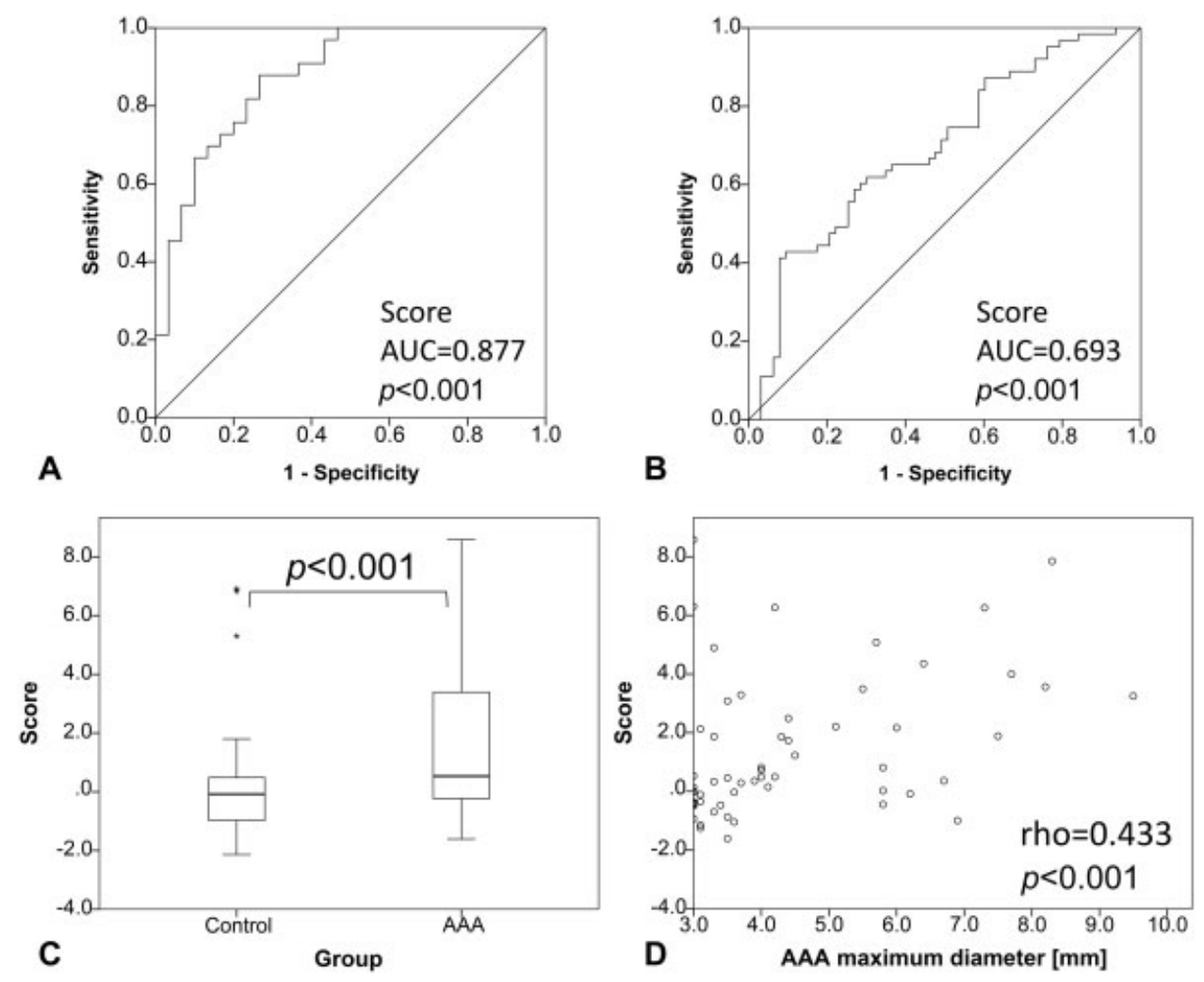

Fig. 4 Diagnostic marker potential of the D-dimer and myeloperoxidase (MPO) based score. Receiver operating characteristics (ROC) curves are given for (A) the initial cohort of abdominal aortic aneurysm (AAA) patients and healthy controls, and (B) the validation cohort. (C) The score distribution is further illustrated by boxplot for the validation set, with a significant difference between AAA patients and controls matched for age, sex and previous cardiovascular disease (Mann-Whitney $U$ test). (D) Score values of the validation cohort are shown in relation to AAA maximum diameter (Spearman's coefficient of correlation). 
Table 6 Correlation of explorative parameters with dimensions of the aneurysm and intraluminal thrombus

\begin{tabular}{|l|l|l|}
\hline Clinical parameter & $R$ & $p$-Value \\
\hline AAA maximum diameter $[\mathrm{mm}]$ & & \\
\hline D-dimer $[\mathrm{\mu g} / \mathrm{mL}]$ & 0.537 & 0.001 \\
\hline MPO $[\mathrm{ng} / \mathrm{mL}]$ & 0.391 & 0.013 \\
\hline Score & 0.664 & $<0.001$ \\
\hline ILT maximum diameter $[\mathrm{mm}]$ & & \\
\hline Newly released neutrophils $\left[\times 10^{6} / \mathrm{L}\right]$ & 0.528 & 0.002 \\
\hline D-dimer $[\mathrm{\mu g} / \mathrm{mL}]$ & 0.500 & 0.009 \\
\hline ILT volume $\left[\mathrm{mm}{ }^{3}\right]$ & & \\
\hline D-dimer $[\mathrm{\mu g} / \mathrm{mL}]$ & 0.687 & $<0.001$ \\
\hline Score & 0.450 & 0.031 \\
\hline
\end{tabular}

Abbreviations: AAA, abdominal aortic aneurysm; ILT, intraluminal thrombus; MPO, myeloperoxidase.

seemed to have a minor impact on the distribution of neutrophil populations.

Despite the fact that the CD16+ monocyte population had only a weak diagnostic marker value for the original collective $(p=0.039)$, reductions in sample size due to confounder matching mostly resulted in $p$-values of $\leq 0.1$ after adjusting for co-morbidities or medication. Yet, matching for aspirin intake, hyperlipidaemia and/or statin therapy as well as hypertension and in particular angiotensin receptor blocker treatment rendered the group difference in CD16+ monocyte frequency insignificant. Comparably, the diagnostic power of the monocyte chemotactic factor CCL2 was lost when the collectives were matched for CHD, hyperlipidaemia, statin or anti-hypertensive therapy.

In contrast, the significant difference in plasma MPO, Ddimer and calculated score was not lost when the AAA and patient collectives were matched for any co-morbidity or medication, even though the MPO marker value was lowered when aspirin or statin therapy were taken into consideration. Of note, MPO, D-dimer and score also correlated with kidney function parameters (Supplementary Table S6, available in the online version), but none of the investigated parameters showed a significant association with the Framingham Risk Score. Finally, when the novel AAA score was evaluated in multivariable analysis by binary logistic regression ( - Table 7), the score as well as hyperlipidaemia and/or lipid-lowering therapy were revealed as significant and independent diagnostic variables, while hypertension and/or anti-hypertensive therapy, CHD and anti-platelet therapy were not.

To validate the score, additional plasma samples of 63 AAA cases and 63 controls were analysed which were independently collected in Leeds (United Kingdom) and one-to-one matched for age, sex and diagnosis of previous cardiovascular disease (composite of: myocardial infarction, angina pectoris, peripheral vascular disease or cerebrovascular disease). In this validation cohort, the AAA and control group differed
Table 7 Multivariable analysis (binary logistic regression) based on 41 AAA cases and 38 controls of the original dataset

\begin{tabular}{|l|l|l|l|l|}
\hline Parameter & Exp(B) & $\begin{array}{l}95 \% \mathrm{Cl} \\
\text { lower } \\
\text { value }\end{array}$ & $\begin{array}{l}95 \% \mathrm{Cl} \\
\text { upper } \\
\text { value }\end{array}$ & $p$-Value \\
\hline Score & 1.873 & 1.085 & 3.234 & 0.024 \\
\hline $\begin{array}{l}\text { Hypertension } \\
\text { and/or anti- } \\
\text { hypertensive } \\
\text { therapy }\end{array}$ & 1.186 & 0.118 & 11.879 & 0.885 \\
\hline $\begin{array}{l}\text { Hyperlipidaemia } \\
\text { and/or lipid- } \\
\text { lowering therapy }\end{array}$ & 13.178 & 2.291 & 75.814 & 0.004 \\
\hline $\begin{array}{l}\text { Coronary heart } \\
\text { disease }\end{array}$ & 1.162 & 0.148 & 9.150 & 0.887 \\
\hline Anti-platelet therapy & 3.410 & 0.580 & 20.035 & 0.175 \\
\hline Constant & 0.085 & & & 0.027 \\
\hline
\end{tabular}

Abbreviation: $\mathrm{Cl}$, confidence interval.

Note: $\operatorname{Exp}(B)$, odds ratio.

significantly $(p<0.001)$ in score values $(-$ Fig. $4 \mathbf{4 C})$ with an AUC of 0.693 in ROC analysis ( - Fig. 4B). In addition, the score correlated positively with maximum aortic diameter ( - Fig. 4D) in the AAA collective $(r=0.433, p<0.001)$ but not in the control group.

\section{The Score Further Predicts Disease Progression}

In a prognostic study design, 33 patients with small aneurysms (without indication for surgical repair; Supplementary Table S7, available in the online version) were followed at 6month intervals for AAA expansion. In 68 datasets, the score was calculated at the beginning of the monitoring period and found to correlate significantly with aneurysm growth over the next 6 months $(r=0.437, p<0.001 ;-$ Fig. 5A). When the events were grouped into slow $(<2 \mathrm{~mm})$ and fast $(\geq 2$ $\mathrm{mm}$ ) progression over 6 months, the score ( $\mathbf{- F i g . 5 B}$ and $\mathbf{C}$ ) outperformed D-dimer (-Fig. 5E and F) in identifying patients with rapid aneurysm progression with an AUC value of $0.701(p=0.006)$ as compared with $0.612(p=0.124)$. A score cut-off at 0.935 was calculated by Youden's index to yield a prognostic sensitivity and specificity of 72 and $67 \%$.

\section{Discussion}

In the present study, we focused on the analysis of circulating monocyte and neutrophil sub-sets and associated plasma factors CCL2 and MPO, evaluating their potential as biomarkers in the context of AAA. We prioritized matching the control group according to sex, age, body mass index and smoking status, with age and smoking being the most important risk factors for AAA. ${ }^{33} \mathrm{~A}$ strong association of plasma D-dimer concentration and AAA has been established for some time and several studies have shown its potential as a diagnostic and prognostic marker. ${ }^{6-8}$ In 

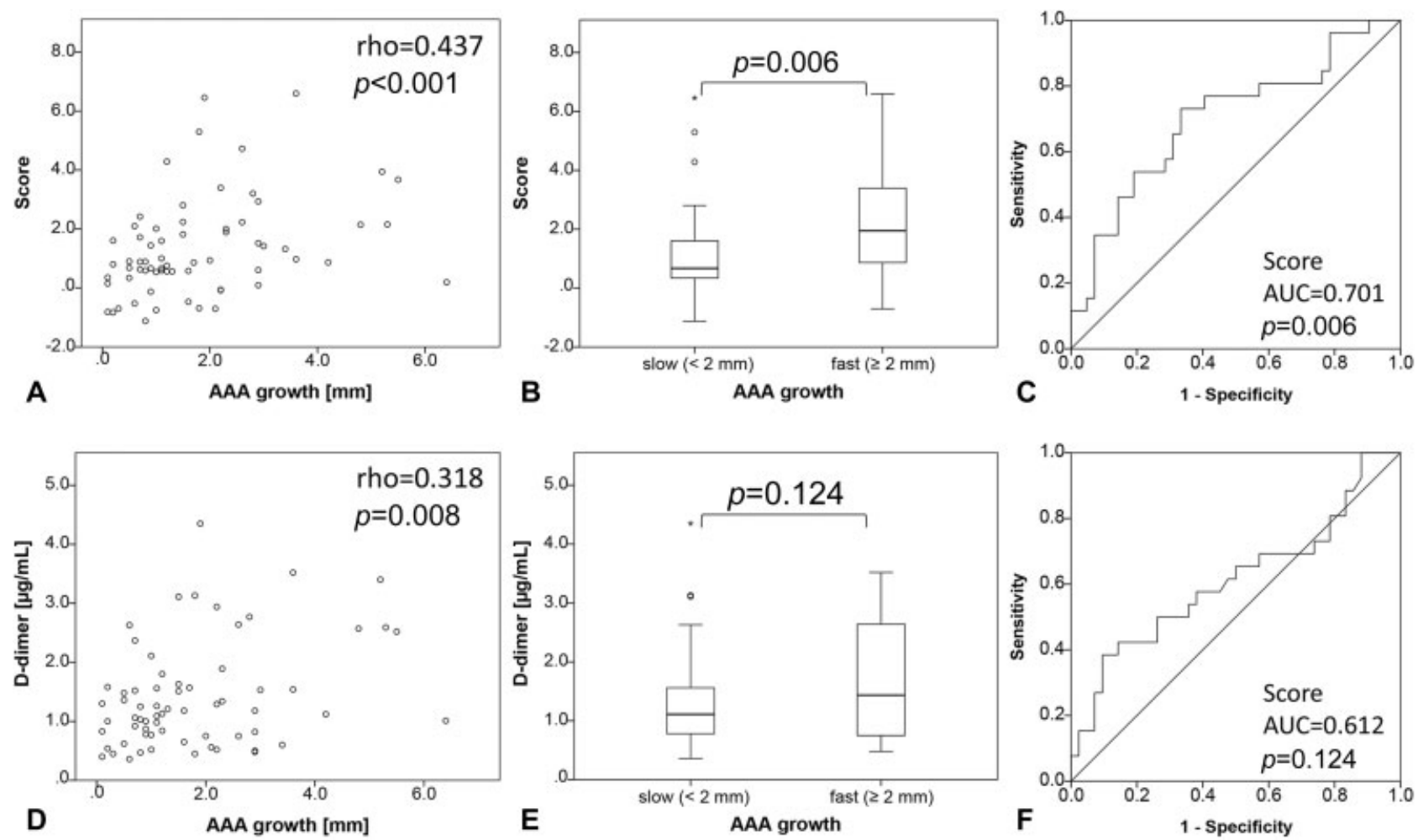

Fig. 5 Prognostic marker potential of the D-dimer and myeloperoxidase (MPO)-based score. The score (A-C) was compared with D-dimer plasma values (D-F) in predicting abdominal aortic aneurysm (AAA) expansion over the next 6 months. (A, D) A direct correlation was assessed by Spearman's test. (B, E) Events were grouped into slow $(<2 \mathrm{~mm})$ and fast $(\geq 2 \mathrm{~mm})$ aneurysm growth in 6 months and evaluated by MannWhitney $U$ test as well as (C, F) receiver operating characteristics (ROC) analysis. Of note, two samples with exceedingly high values for D-dimer $(15.3$ and $24.3 \mu \mathrm{g} / \mathrm{mL}$ ) and the calculated score (21.0 and 37.9) were omitted from the graphs $\mathrm{A}, \mathrm{B}, \mathrm{D}$ and $\mathrm{E}$ to improve resolution.

agreement with these reports, the D-dimer levels were 2.8fold increased in our patient group and showed a significant correlation with AAA maximum diameter and markedly with ILT volume.

The chronic inflammation and in particular the infiltration by myeloid cell populations in ILT and AAA wall are considered central elements of AAA pathogenesis. ${ }^{34,35}$ We hypothesized that changes in the activation state of monocytes and neutrophils might also be observed in the systemic circulation of AAA patients. Both chronic and acute inflammatory states are often accompanied by a selective increase in the CD16+ monocyte sub-sets. ${ }^{17,36} \mathrm{~A}$ rise in the frequency of CD16 expressing monocytes was also found in our AAA patients (18.8\%) versus controls (16.0\%) and is in agreement with previously published results by Ghigliotti et $\mathrm{al}^{23}$ who reported 15.1 and $11.0 \%$, respectively. It should be noted that the difference in recorded values may relate to distinct flow cytometric approaches to eliminate 'contaminating' lymphocytes and granulocytes in monocyte detection. However, we found the diagnostic power of CD16+ monocytes for AAA (AUC $=0.646, p=0.039)$ to be rather limited when compared with other myeloid cell activation markers.

Considering the neutrophil sub-sets, we based our methodological approach on the previously published marker selection by Pillay et al..$^{25}$ To exclude interference by transient inflammatory states, blood collection was postponed if patients had a known infection. A significantly increased number of activated and newly released neutrophils was recorded in the AAA patient versus control group. This likely reflects the AAA site of chronic inflammation and immune cell recruitment.

In the further investigation, we followed the notion that systemically observed cellular activation and recruitment of myeloid cells might be accompanied by a measurable change in plasma cytokine or chemokine levels. Thus, an initial screening of 10 patient versus 10 control samples was performed using a multiplex bead array for several factors known to influence or reflect myeloid cell activation, differentiation and migration. While most of the investigated cytokines and chemokines had previously been detected in AAA tissue, ${ }^{37}$ limited information was available on their blood values in AAA patients. ${ }^{38}$ In our reduced collective, only MPO and CCL2 differed significantly between the two study groups which was subsequently confirmed by ELISA. Elevations of other factors might be detected with a larger sample size. However, the increase of plasma MPO and CCL2 in AAA patients (compared with healthy controls) proved to be most pronounced and hence exhibit the best biomarker potential. CCL2 is a chemoattractant for monocytes, ${ }^{39}$ a differentiation stimulus enhancing CD16 expression on monocytes ${ }^{40}$ and drives a vicious circle of inflammation and SMC apoptosis. ${ }^{41,42}$ MPO has received little research 
attention in the AAA context with differential results regarding its marker potential. ${ }^{8,43}$ It is of central importance for the innate defence catalyzing the formation of highly bacteriotoxic hypochlorite radical species, but is also known to contribute to media destruction in AAA. ${ }^{34}$ In our study, we found moderately elevated plasma levels of CCL2 but highly increased (in median doubled) MPO blood concentrations in the patient group. Moreover, plasma MPO correlated with tissue-released MPO and with maximum AAA diameter, identifying the aneurysm as the primary source for circulating MPO. Of note, several patients with an aortic diameter beyond $80 \mathrm{~mm}$ had moderate MPO blood concentrations. This might indicate that in very large, non-ruptured aneurysms the balance tilts towards fibrotic rather than inflammatory processes. The superior marker potential of plasma MPO was further confirmed by ROC analysis and may relate to the circumstance that MPO is released by both, activated neutrophils as well as CD16+ monocytes. ${ }^{44,45}$

In multivariable logistic regression models including the investigated myeloid cell parameters, MPO prevailed as an independent marker for AAA diagnosis along with the established AAA marker, D-dimer. The two parameters may be combined in a diagnostic score reaching a sensitivity of $73 \%$ and specificity of $80 \%$ with the cut-off set to 0 , and independent of age, sex and smoker status. To address the issue of potential confounders not considered when matching patient and control collectives, the devised diagnostic score and all explorative parameters, which had been found to differ significantly between the groups, were re-evaluated after adjustment for co-morbidities or stable medication. The two collectives differed most prominently in hyperlipidaemia, statin use and aspirin therapy, but also in the frequency of CHD, hypertension and in kidney function. Overall, the neutrophil and monocyte sub-sets as well as CCL2, which had moderate diagnostic marker value, were more sensitive to confounders. In contrast, plasma MPO, D-dimer and the deduced score proved significantly different between the collectives even after adjustment for confounding conditions, and the score prevailed in multivariable analysis as an independent diagnostic variable. A further indication of the robustness of the diagnostic score was provided by a validation set of 126 independently collected plasma samples from AAA patients and controls matched for sex, age and previous cardiovascular disease.

Importantly, regarding the monitoring of patients with a known AAA diagnosis, the score also showed prognostic marker value which exceeded the potency of D-dimer alone. Based on a cut-off set to 0.935 , the score could identify patients with rapid progression ( $\geq 2 \mathrm{~mm}$ over the next 6 months) with $72 \%$ sensitivity and $67 \%$ specificity. Thus, the score holds an important potential for patient stratification.

In conclusion, our data provide proof-of-concept that myeloid inflammation markers constitute a circulating footprint of atherosclerotic aortic disease. We have provided first evidence that a combined score of plasma MPO and D-dimer may prove beneficial in AAA diagnosis and prognosis. While D-dimer is a confirmed and robust, albeit non-specific AAA indicator, it is primarily associated with the presence and size of the ILT. By including MPO as a second parameter related to the central pathophysiological component of inflammation, the deduced score is more closely associated with AAA diameter than D-dimer and holds the potential to also detect and monitor aneurysms with no or little thrombus. The diagnostic application of the score may be limited to countries without ultrasound screening program and requires further large-scale validation with unmatched cohorts. The prognostic value of the score to predict disease progression seems to be of particular interest as it may serve clinicians to better guide the timing of therapeutic interventions and would thus merit evaluation in prospective multicentre studies.

\section{Limitations of the Study}

The sample size of our study has certainly limited the detection of potential biomarkers to parameters which show a substantial difference in mean values between groups and/or small biological variance. While this may prove beneficial in clinical practice, it does not reflect the parameter importance in AAA pathogenesis and may also explain discordances between our data and published literature.

As the study was explorative in design, no correction for multiple testing was applied which increases the risk of committing a type I error. However, the deduced diagnostic score was subsequently challenged with a validation cohort and proved significant irrespective of cardiovascular disease.

D-dimer and MPO assessment can be included in routine blood analysis at a cost of $€ 83$ for both tests ( $€ 24$ for D-dimer, $€ 59$ for MPO) currently charged by the local routine laboratory. D-dimer measurements are generally robust, based on citrated plasma and widely offered by routine laboratories. In contrast, MPO assays are less frequent in clinical use, but standardized tests are available for validated general chemistry analysers (e.g. Diazyme Latex Enhanced Immunoturbidimetric MPO Assay). While these assays are offered for standard ethylenediaminetetraacetic acid or heparin plasma, a more careful pre-analytical sample handling (such as blood storage on ice, centrifugation within the hour, as conducted in our study) may be required to prevent artificial myeloid cell activation, enable accurate plasma MPO assessment and score calculation and will thus have to be further evaluated for clinical routine application.

With respect to leukocyte sub-set measurement, a single platform approach with absolute counting beads would have provided greater precision with a potentially better diagnostic value for neutrophil and monocyte sub-sets than detected in the current study. Furthermore, the apoptotic rate of myeloid cells was not addressed in our study (since dead cells were excluded from the analysis by 7-AAD staining). However, the increased numbers of activated neutrophils which are reportedly exhibiting delayed apoptosis 46 and of newly released neutrophils recruited upon neutrophil loss may indirectly reflect changes in the balance of the neutrophil apoptotic rate. 


\section{What is known about this topic?}

- The abdominal aortic aneurysm (AAA) is mostly asymptomatic and hence difficult to diagnose unless routine ultrasound screening is implemented in the national health plan. Furthermore, the prediction of rapid AAA progression is vital, as it is associated with a high risk of rupture and patient death.

- The best characterized diagnostic and prognostic blood parameter to date is the fibrin degradation product D-dimer which is related to the frequently occurring intraluminal thrombus but is also increased in other cardiovascular disorders.

- Pre-clinical models have shown a central role for neutrophils and monocytes in AAA pathogenesis which points to a potential of myeloid factors as AAA biomarkers.

\section{What does this paper add?}

- Based on a comprehensive comparison of neutrophil and monocyte activation parameters, myeloperoxidase was identified as the most elevated myeloid marker in AAA patient blood.

- D-dimer and myeloperoxidase represent two sensitive biomarkers of AAA which reflect distinct components of the AAA pathomechanism and when combined yield an improved score for AAA diagnosis and, more importantly, for prediction of rapid progression.

\section{Funding}

This work was primarily supported by the Austrian Science Fund (SFB project F 5409-B21) as well as the Medical Scientific Fund of the Mayor of the City of Vienna (project 15012) and The Garfield Weston Foundation (for the Leeds Aneurysm Development Study). Marc Bailey is supported by the British Heart Foundation. The sponsors had no role in study design; in the collection, analysis and interpretation of data; in the writing of the report; nor in the decision to submit the article for publication.

\section{Conflict of Interest}

None declared.

\section{Acknowledgements}

We would like to thank Edith Lackner, Christa Drucker and Christian Schoergenhofer for providing samples from the human endotoxaemia study for assay validation purposes, and Fraser MacRae for sample preparation in Leeds. Furthermore, we thank Prof. Georg Heinze (Centre for Medical Statistics, Informatics, and Intelligent Systems, Medical University of Vienna), Mateusz Ruks and Agota Wunderli for their support in developing logistic regression models.

\section{References}

1 Moll FL, Powell JT, Fraedrich G, et al; European Society for Vascular Surgery. Management of abdominal aortic aneurysms clinical practice guidelines of the European society for vascular surgery. Eur J Vasc Endovasc Surg 2011;41(Suppl 1):S1-S58

2 Takagi H, Manabe H, Kawai N, Goto S, Umemoto T. Plasma fibrinogen and D-dimer concentrations are associated with the presence of abdominal aortic aneurysm: a systematic review and meta-analysis. Eur J Vasc Endovasc Surg 2009;38(03):273-277

3 Sidloff DA, Stather PW, Choke E, Bown MJ, Sayers RD. A systematic review and meta-analysis of the association between markers of hemostasis and abdominal aortic aneurysm presence and size. J Vasc Surg 2014;59(02):528-535

4 De Haro J, Acin F, Bleda S, Varela C, Medina FJ, Esparza L. Prediction of asymptomatic abdominal aortic aneurysm expansion by means of rate of variation of C-reactive protein plasma levels. J Vasc Surg 2012;56(01):45-52

5 Lee AJ, Fowkes FG, Lowe GD, Rumley A. Haemostatic factors, atherosclerosis and risk of abdominal aortic aneurysm. Blood Coagul Fibrinolysis 1996;7(07):695-701

6 Vele E, Kurtcehajic A, Zerem E, Maskovic J, Alibegovic E, Hujdurovic A. Plasma D-dimer as a predictor of the progression of abdominal aortic aneurysm. J Thromb Haemost 2016;14(11): 2298-2303

7 Golledge J, Muller R, Clancy P, McCann M, Norman PE. Evaluation of the diagnostic and prognostic value of plasma D-dimer for abdominal aortic aneurysm. Eur Heart J 2011;32(03): 354-364

8 Vega de Ceniga M, Esteban M, Barba A, Estallo L, Blanco-Colio LM, Martin-Ventura JL. Assessment of biomarkers and predictive model for short-term prospective abdominal aortic aneurysm growth-a pilot study. Ann Vasc Surg 2014;28(07):1642-1648

9 Hellenthal FAMVI, Geenen ILA, Teijink JAW, Heeneman S, Schurink GW. Histological features of human abdominal aortic aneurysm are not related to clinical characteristics. Cardiovasc Pathol 2009;18(05):286-293

10 Houard X, Touat Z, Ollivier V, et al. Mediators of neutrophil recruitment in human abdominal aortic aneurysms. Cardiovasc Res 2009;82(03):532-541

11 Qin Y, Cao X, Yang Y, Shi GP. Cysteine protease cathepsins and matrix metalloproteinases in the development of abdominal aortic aneurysms. Future Cardiol 2013;9(01):89-103

12 Ramos-Mozo P, Madrigal-Matute J, Vega de Ceniga M, et al. Increased plasma levels of NGAL, a marker of neutrophil activation, in patients with abdominal aortic aneurysm. Atherosclerosis 2012;220(02):552-556

13 Juvonen J, Surcel HM, Satta J, et al. Elevated circulating levels of inflammatory cytokines in patients with abdominal aortic aneurysm. Arterioscler Thromb Vasc Biol 1997;17(11):2843-2847

14 Ziegler-Heitbrock L, Ancuta P, Crowe S, et al. Nomenclature of monocytes and dendritic cells in blood. Blood 2010;116(16): e74-e80

15 Patel AA, Zhang Y, Fullerton JN, et al. The fate and lifespan of human monocyte subsets in steady state and systemic inflammation. J Exp Med 2017;214(07):1913-1923

16 Wong KL, Tai JJ, Wong WC, et al. Gene expression profiling reveals the defining features of the classical, intermediate, and nonclassical human monocyte subsets. Blood 2011;118(05):e16-e31

17 Zawada AM, Rogacev KS, Rotter B, et al. SuperSAGE evidence for CD14++CD16+ monocytes as a third monocyte subset. Blood 2011;118(12):e50-e61

18 Chimen M, Yates CM, McGettrick HM, et al. Monocyte subsets coregulate inflammatory responses by integrated signaling through TNF and IL-6 at the endothelial cell interface. J Immunol 2017;198(07):2834-2843

19 Auffray C, Fogg D, Garfa M, et al. Monitoring of blood vessels and tissues by a population of monocytes with patrolling behavior. Science 2007;317(5838):666-670 
20 Cros J, Cagnard N, Woollard K, et al. Human CD14dim monocytes patrol and sense nucleic acids and viruses via TLR7 and TLR8 receptors. Immunity 2010;33(03):375-386

21 Shantsila E, Lip GY. The role of monocytes in thrombotic disorders. Insights from tissue factor, monocyte-platelet aggregates and novel mechanisms. Thromb Haemost 2009;102(05):916-924

22 Rogacev KS, Cremers B, Zawada AM, et al. CD14++CD16+ monocytes independently predict cardiovascular events: a cohort study of 951 patients referred for elective coronary angiography. J Am Coll Cardiol 2012;60(16):1512-1520

23 Ghigliotti G, Barisione C, Garibaldi S, et al. CD16(+) monocyte subsets are increased in large abdominal aortic aneurysms and are differentially related with circulating and cell-associated biochemical and inflammatory biomarkers. Dis Markers 2013; 34(02):131-142

24 Rubio-Navarro A, Amaro Villalobos JM, Lindholt JS, et al. Hemoglobin induces monocyte recruitment and CD163-macrophage polarization in abdominal aortic aneurysm. Int J Cardiol 2015; 201:66-78

25 Pillay J, Ramakers BP, Kamp VM, et al. Functional heterogeneity and differential priming of circulating neutrophils in human experimental endotoxemia. J Leukoc Biol 2010;88(01):211-220

26 Kamp VM, Pillay J, Lammers JWJ, Pickkers P, Ulfman LH, Koenderman L. Human suppressive neutrophils CD16bright/CD62Ldim exhibit decreased adhesion. J Leukoc Biol 2012;92(05): 1011-1020

27 Al-Barjas HS, Ariëns R, Grant P, Scott JA. Raised plasma fibrinogen concentration in patients with abdominal aortic aneurysm. Angiology 2006;57(05):607-614

28 Bailey MA, Griffin KJ, Sohrabi S, et al. Plasma thrombin-antithrombin complex, prothrombin fragments 1 and 2, and D-dimer levels are elevated after endovascular but not open repair of infrarenal abdominal aortic aneurysm. J Vasc Surg 2013;57(06): $1512-1518$

29 Parry DJ, Al-Barjas HS, Chappell L, Rashid ST, Ariëns RA, Scott DJ. Markers of inflammation in men with small abdominal aortic aneurysm. J Vasc Surg 2010;52(01):145-151

30 Scott DJ, Prasad P, Philippou H, et al. Clot architecture is altered in abdominal aortic aneurysms and correlates with aneurysm size. Arterioscler Thromb Vasc Biol 2011;31(12):3004-3010

31 Sohrabi S, Wheatcroft S, Barth JH, et al. Cardiovascular risk in patients with small and medium abdominal aortic aneurysms, and no history of cardiovascular disease. Br J Surg 2014;101(10): $1238-1243$

32 Tjur T. Coefficients of determination in logistic regression models -a new proposal: the coefficient of discrimination. Am Stat 2009; 63:366-372

33 Vardulaki KA, Walker NM, Day NE, Duffy SW, Ashton HA, Scott RA. Quantifying the risks of hypertension, age, sex and smoking in patients with abdominal aortic aneurysm. Br J Surg 2000;87(02): 195-200

34 Piechota-Polanczyk A, Jozkowicz A, Nowak W, et al. The abdominal aortic aneurysm and intraluminal thrombus: current concepts of development and treatment. Front Cardiovasc Med 2015;2:19

35 Dale MA, Ruhlman MK, Baxter BT. Inflammatory cell phenotypes in AAAs: their role and potential as targets for therapy. Arterioscler Thromb Vasc Biol 2015;35(08):1746-1755

36 Ancuta P, Wang J, Gabuzda D. CD16+ monocytes produce IL-6, CCL2, and matrix metalloproteinase-9 upon interaction with CX3CL1-expressing endothelial cells. J Leukoc Biol 2006;80(05): 1156-1164

37 Middleton RK, Lloyd GM, Bown MJ, Cooper NJ, London NJ, Sayers RD. The pro-inflammatory and chemotactic cytokine microenvironment of the abdominal aortic aneurysm wall: a protein array study. J Vasc Surg 2007;45(03):574-580

38 Jones GT, Phillips LV, Williams MJ, van Rij AM, Kabir TD. Two C-C family chemokines, eotaxin and RANTES, are novel independent plasma biomarkers for abdominal aortic aneurysm. J Am Heart Assoc 2016;5(05):5

39 Mayr FB, Spiel AO, Leitner JM, et al. Influence of the Duffy antigen on pharmacokinetics and pharmacodynamics of recombinant monocyte chemoattractant protein (MCP-1, CCL-2) in vivo. Int J Immunopathol Pharmacol 2009;22(03):615-625

40 Feng AL, Zhu JK, Sun JT, et al. CD16+ monocytes in breast cancer patients: expanded by monocyte chemoattractant protein- 1 and may be useful for early diagnosis. Clin Exp Immunol 2011;164 (01):57-65

41 Yamanouchi D, Morgan S, Kato K, Lengfeld J, Zhang F, Liu B. Effects of caspase inhibitor on angiotensin II-induced abdominal aortic aneurysm in apolipoprotein E-deficient mice. Arterioscler Thromb Vasc Biol 2010;30(04):702-707

42 Wang Q Ren J, Morgan S, Liu Z, Dou C, Liu B. Monocyte chemoattractant protein-1 (MCP-1) regulates macrophage cytotoxicity in abdominal aortic aneurysm. PLoS One 2014;9(03):e92053

43 Pradhan-Palikhe P, Vikatmaa P, Lajunen T, et al. Elevated MMP-8 and decreased myeloperoxidase concentrations associate significantly with the risk for peripheral atherosclerosis disease and abdominal aortic aneurysm. Scand J Immunol 2010;72(02):150-157

44 Odobasic D, Kitching AR, Holdsworth SR. Neutrophil-mediated regulation of innate and adaptive immunity: the role of myeloperoxidase. J Immunol Res 2016;2016:2349817

45 Wildgruber M, Aschenbrenner T, Wendorff H, et al. The "Intermediate" CD14 ${ }^{++} \mathrm{CD} 16^{+}$monocyte subset increases in severe peripheral artery disease in humans. Sci Rep 2016;6:39483

46 Choi M, Rolle S, Wellner M, et al. Inhibition of NF-kappaB by a TATNEMO-binding domain peptide accelerates constitutive apoptosis and abrogates LPS-delayed neutrophil apoptosis. Blood 2003; 102(06):2259-2267 